\title{
Asymptotic Behavior of the coupled Klein-Gordon-Schrödinger systems on compact manifolds
}

\author{
César Augusto Bortot ${ }^{1}$, Thales Maier Souza ${ }^{1}$, and Janaina Zanchetta ${ }^{2}$ \\ ${ }^{1}$ Universidade Federal de Santa Catarina \\ ${ }^{2}$ Universidade Estadual de Maringa
}

May 4, 2021

\begin{abstract}
This paper is concerned with a 2-dimensional Klein-Gordon-Schrödinger system subject to two types of locally distributed damping on a compact Riemannian manifold $\$ \backslash$ mathcal $\{M\} \$$ without boundary. Making use of unique continuation property, the observability inequalities, and the smoothing effect due to Aloui, we obtain exponential stability results.
\end{abstract}

\section{Hosted file}

KGS 2021.pdf available at https://authorea.com/users/411730/articles/520651-asymptoticbehavior-of-the-coupled-klein-gordon-schr\%C3\%B6dinger-systems-on-compact-manifolds 